\title{
ON CONGRUENCES OF DOUBLE $p$-ALGEBRAS WITH NONVOID CORE
}

\author{
by V. KOUBEK and J. SICHLER
}

(Received 9 June, 1992)

1. Introduction. An algebra $L=\left(A ; \vee, \wedge,{ }^{*},{ }^{+}, 0,1\right)$ of type $(2,2,1,1,0,0)$ is called a distributive double p-algebra whenever its reduct $(A, \vee, \wedge, 0,1)$ is a distributive $(0,1)$-lattice that, for any $a \in A$, contains a greatest element $a^{*}$ such that $a \wedge a^{*}=0$ and a least element $a^{+}$for which $a \vee a^{+}=1$.

According to [2], any distributive double $p$-algebra $L$ can be extended to a congruence permutable algebra $K$ in such a way that every congruence on $L$ has a unique extension to $K$. Since distributive double $p$-algebras enjoy the congruence extension property, see Katriňák [4], it follows that the congruence lattice Con $(L)$ of any distributive double $p$-algebra $L$ is isomorphic to the congruence lattice of a distributive double $p$-algebra $K$ whose congruences permute.

In [1], Adams and Beazer demonstrated that the congruence permutability of $K$ is equivalent to the absence of 4-element chains in its poset $P(K)$ of prime ideals. The present note grew from an attempt to construct an extension $K$ of a distributive double $p$-algebra $L$ with the unique congruence extension property whose maximal prime ideal chains have more uniform lengths and, in particular, from the question about the existence of extensions with no singleton maximal prime ideal chains. Such algebras are exactly those with a core element $k$, that is, an element such that $k^{*}=0$ and $k^{+}=1$.

The present note shows that such extensions do not always exist and describes those algebras for which they do.

To formulate our main result, Theorem 1.1, we need the following notions.

For any distributive double $p$-algebra $L$, the set $\psi$ of all pairs $(a, b) \in L^{2}$ satisfying $a^{*}=b^{*}$ is a congruence of the $p$-algebra reduct $\left(A ; \vee, \wedge,{ }^{*}, 0,1\right)$, and the dually defined relation $\psi_{+}$is a congruence of $\left(A ; \vee, \wedge,{ }^{+}, 0,1\right)$. Clearly, the meet $\Phi=\psi_{*} \wedge \psi_{+}$is the determination congruence $\Phi \in \operatorname{Con}(L)$. It will be shown that the equivalence $\Psi=\psi_{*} \vee$ $\psi_{+}$is a member of $\operatorname{Con}(L)$, namely the least congruence $\Theta$ of $L$ for which the quotient $L / \Theta$ is a Boolean algebra. Since $\left(a^{*}, a^{+}\right) \in \Psi$ for every $a \in L$, the congruence $\Psi$ is total for any algebra whose core is nonvoid.

In Theorem 1.1 below, the condition (2) is a reformulation of (3) in terms of the Priestley dual $X$ of $L$; Priestley duality will be briefly reviewed in the second section and used throughout the paper.

TheOREM 1.1. Let $L$ be a distributive double p-algebra and let $X$ be its Priestley dual. Then the following properties are equivalent:

(1) $L$ has an extension $K$ with nonvoid core such that every congruence of $L$ uniquely extends to a congruence of $K$;

(2) $X$ has a decomposition $X=V_{0} \cup V_{1} \cup N(X)$, where $V_{0} \supseteq \operatorname{Min}(X) \backslash \operatorname{Max}(X)$ and $V_{1} \supseteq \operatorname{Max}(X) \backslash \operatorname{Min}(X)$ are open and $N(X)=\operatorname{Max}(X) \cap \operatorname{Min}(X)$;

(3) there exist lattice congruences $\psi_{0} \leq \psi_{*}$ and $\psi_{1} \leq \psi_{+}$such that $\psi_{0} \vee \psi_{1}=\psi_{*} \vee$ $\psi_{+}=\Psi$ and $\psi_{0} \wedge \psi_{1}=\omega$, the least congruence of $L$.

Furthermore, if $L$ satisfies any of these conditions, then $K$ is congruence permutable.

Glasgow Math. J. 35 (1993) 385-394. 
Let $L$ be a regular double $p$-algebra, that is, an algebra from the variety $\mathbf{R}$ determined by the identity equivalent to $x \wedge x^{+} \leq y \vee y^{*}$. An algebra $L$ is regular exactly when all chains of its prime ideals have at most two elements or, equivalently, when every congruence of $L$ is regular, that is, fully determined by any of its classes, see Varlet [9]. Since the determination congruence $\Phi$ of any regular algebra is trivial, the choice of $\psi_{0}{ }^{*}=\psi_{*}$ and $\psi_{1}=\psi_{+}$satisfies (3) of Theorem 1.1 , and we immediately obtain the first claim of the following result.

Corollary 1.2. Any regular double p-algebra $L$ has an extension $K$ with nonvoid core such that every congruence of $L$ uniquely extends to a congruence of $K$. Moreover, $K$ is a regular algebra.

In other words, in the regular case, all maximal prime ideal chains of $K$ have two elements and all congruences of $K$ permute.

For any element $x$ in a double $p$-algebra, define $x^{(0 *+)}=x$ and, for any integer $n \geq 0$, $x^{(n+1)(*+)}=\left(x^{n(*+)}\right)^{*+}$. A variety $\mathbf{V}$ of distributive double $p$-algebras is of finite range $n \geq 0$ if it satisfies the identity $x^{n(*+)}=x^{(n+1)(*+)}$. It is well known that every nonregular variety of a finite range $n$ contains the variety $\mathbf{S}$ of double Stone algebras, that is, the variety generated by the 4-element chain algebra.

Following a brief review of Priestley duality, we prove Theorem 1.1 and conclude with some examples of double Stone algebras that do not satisfy the extendability conditions of Theorem 1.1. This will show that every nonregular variety $\mathbf{V}$ of finite range fails to satisfy a varietal analogue of Corollary 1.2.

2. Preliminaries. Let $(X, \tau, \leq)$ be an ordered topological space and let $Y \subseteq X$. We write $(Y]=\{x \in X \mid x \leq y$ for some $y \in Y\}$ and say that $Y$ is decreasing if $(Y]=Y$. Dually, we set $[Y]=\{x \in X \mid y \leq x$ for some $y \in Y\}$ and say that $Y$ is increasing if $[Y)=Y$. If $\operatorname{Max}(X)$ and $\operatorname{Min}(X)$ are the respective sets of all maximal or minimal elements of $(X, \tau, \leq)$, we write

$\operatorname{Ext}(X)=\operatorname{Max}(X) \cup \operatorname{Min}(X), \operatorname{Mid}(X)=X \backslash \operatorname{Ext}(X)$ and $N(X)=\operatorname{Max}(X) \cap \operatorname{Min}(X)$.

For any $Y \subseteq X$ we set $\operatorname{Max}(Y)=[Y) \cap \operatorname{Max}(X), \quad \operatorname{Min}(Y)=(Y] \cap \operatorname{Min}(X)$ and $\operatorname{Ext}(Y)=\operatorname{Max}(Y) \cup \operatorname{Min}(Y)$. For $Y=\{x\}$, we write $\operatorname{Max}(x)$ instead of $\operatorname{Max}(Y)$, etc.

A compact ordered space $(X, \tau, \leq)$ is called a Priestley space whenever it is totally order disconnected, that is, such that for any $x \neq y$ there is a clopen decreasing $C \subset X$ with $y \in C$ and $x \notin C$. Any Priestley space has the following separation property (cf. also [5]):

(S) if $Y, Z \subseteq X$ are closed and such that $Y \cap(Z]=\varnothing$ then $(Z] \subseteq D$ and $Y \cap D=\varnothing$ for some clopen decreasing $D \subset X$.

The category $\mathbf{P}$ of all continuous order preserving maps between Priestley spaces is tied to the category $\mathbf{D}$ of all $(0,1)$-homomorphisms of distributive $(0,1)$-lattices by contravariant functors $D: \mathbf{P} \rightarrow \mathbf{D}$ and $P: \mathbf{D} \rightarrow \mathbf{P}$ whose composites $D \circ P$ and $P \circ D$ are naturally equivalent to the identity functors of their respective domains, see Priestley [6], [7]. This pair of functors constitutes the Priestley duality. In this duality, for any object $D$ of $\mathbf{D}$, the Priestley space $P(D)$ consists of the prime ideals of $D$, ordered by inclusion and suitably topologized, while $D(P) \in \mathbf{D}$ is the set of all clopen decreasing sets of the Priestley space $P$. Furthermore, surjective $\mathbf{P}$-morphisms represent one-to-one $(0,1)$ homomorphisms, while each lattice congruence $\psi$ of $D$ is represented by a closed 
subspace $F \subseteq P(D)$ so that $(a, b) \in \psi$ if and only if the clopen subsets $A, B$ of $P(D)$ corresponding to $a, b$, respectively, satisfy $A \cap F=B \cap F$, see Priestley [8].

We also recall that, for every $x \in X \in \mathbf{P}$, the sets $\operatorname{Max}(x)$ and $\operatorname{Min}(x)$ are nonvoid.

In [8] it is shown that Priestley duals of distributive double $p$-algebras, called $d p$-spaces here, are exactly the Priestley spaces $(X, \tau, \leq)$ such that

(D) the set $[D)$ is clopen for any clopen decreasing $D \subseteq X$, and (E] is clopen whenever $E \subseteq X$ is clopen and increasing.

Thus if $X=(X, \tau, \leq)$ is a $d p$-space and if a clopen decreasing set $A \subseteq X$ represents $a \in D(X)$, then $a^{*}$ is represented by the clopen decreasing set $X \backslash[A)=X \backslash[\operatorname{Min}(A))$, while $a^{+}$corresponds to the clopen decreasing set $(X \backslash A]=(\operatorname{Max}(X) \backslash A]$. Since the subsets $\operatorname{Max}(X)$ and $\operatorname{Min}(X)$ of any $d p$-space $X$ are closed, they represent lattice congruences on $L$; it is easy to see that $\operatorname{Min}(X)$ represents $\psi_{0}$ while $\operatorname{Max}(X)$ represents $\psi_{1}$.

According to $[8]$, a continuous order preserving mapping $f:(X, \tau, \leq) \rightarrow(Y, \sigma, \leq)$ between $d p$-spaces is the Priestley dual of a morphism of the corresponding distributive double $p$-algebras if and only if

$$
f(\operatorname{Max}(x))=\operatorname{Max}(f(x)) \text { and } f(\operatorname{Min}(x))=\operatorname{Min}(f(x)) \text { for every } x \in X .
$$

An alternative description of $d p$-spaces will be useful.

Lemma 2.1. The following are equivalent for any Priestley space $(X, \tau, \leq)$ :

(1) $(X, \tau, \leq)$ is a dp-space;

(2) $\operatorname{Min}(\bar{S}) \subseteq \overline{\operatorname{Min}(S)}$ and $\operatorname{Max}(\bar{S}) \subseteq \overline{\operatorname{Max}(S)}$ for every $S \subseteq X$;

(3) $\operatorname{Min}(\bar{S})=\overline{\operatorname{Min}(S)}$ and $\operatorname{Max}(\bar{S})=\overline{\operatorname{Max}(S)}$ for every $S \subseteq X$.

Proof. Assume (1). To prove the first claim of (2), let $S \subseteq X$ be arbitrary and $x \in \bar{S}$; we need only show that $\operatorname{Min}(x) \subseteq \overline{\operatorname{Min}(S)}$.

If $m \in \operatorname{Min}(x) \backslash \overline{\operatorname{Min}(S)}$, then $(m]=\{m\}$ is disjoint with the closed set $\overline{\operatorname{Min}(S)}$, so that there exists a clopen decreasing set $C$ such that $m \in C$ and $C \cap \overline{\operatorname{Min}(S)}=\varnothing$. Since $X$ is a $d p$-space, the set $[C]$ is open and, clearly, $x \in[C)$. But then $x \in \bar{S}$ implies the existence of elements $c \in C$ and $s \in S$ such that $c \leq s$, and hence $\operatorname{Min}(c) \subseteq \operatorname{Min}(s)$. Since $C$ is decreasing, the nonvoid set $\operatorname{Min}(c)$ is contained in $C$ and, consequently, $C \cap \overline{\operatorname{Min}(S)} \supseteq$ $C \cap \operatorname{Min}(s) \supseteq \operatorname{Min}(c) \neq \varnothing$, in contradiction to the choice of $C$. Since the proof of the second part of (2) is dual, this demonstrates (2).

To prove that (2) implies (1), let $A \subseteq X$ be clopen and decreasing. We must show that $S=X \backslash[A)$ is closed. First we note that

$$
S=\{x \in X \mid \operatorname{Min}(x) \cap A=\varnothing\},
$$

because $A$ is decreasing. The set $S$ is decreasing, so that $\operatorname{Min}(s) \subseteq S$ for every $s \in S$; but then (Q) implies that $\operatorname{Min}(S) \subseteq X \backslash A$. The set $X \backslash A$ is closed and, by $(2), \operatorname{Min}(\bar{S}) \subseteq$ $\overline{\operatorname{Min}(S)} \subseteq X \backslash A$. For any $x \in \bar{S}$, we then have $\operatorname{Min}(x) \cap A=\varnothing$, and hence $x \in S$ by (Q). Therefore $\bar{S}=S$ as claimed. Together with a dual argument, this implies (1).

Obviously, (3) implies (2).

Since $\operatorname{Min}(X)$ is closed in any $d p$-space $X$, the set $\operatorname{Min}(\bar{S})=(\bar{S}] \cap \operatorname{Min}(X)$ is closed and contains $\operatorname{Min}(S)$, so that $\overline{\operatorname{Min}(S)} \subseteq \operatorname{Min}(\bar{S})$ as well. Together with the first section of the proof and its dual, this shows that (1) implies (3).

The following result of [2] will be needed. 
THEOREM 2.2, [2]. For every distributive double p-algebra $L$ there exists a congruence permutable algebra $L^{\prime}$ containing $L$ such that every congruence of $L$ extends uniquely to $L^{\prime}$. The Priestley dual $g: X^{\prime} \rightarrow X$ of the inclusion $L \subseteq L^{\prime}$ is the identity map and the subposet $\operatorname{Mid}\left(X^{\prime}\right)$ of $X^{\prime}$ is an antichain.

From [3] we recall that congruences of any distributive double $p$-algebra $L$ correspond to those closed subsets $Z$ of the Priestley dual $X=P(L)$ that satisfy $\operatorname{Min}(z) \subseteq Z$ and $\operatorname{Max}(z) \subseteq Z$ for every $z \in Z$. Following Davey [3], we call any such $Z$ a closed $c$-set or simply a cc-set.

Thus, for instance, the $c c$-set $\operatorname{Ext}(X)=\operatorname{Max}(X) \cup \operatorname{Min}(X)$ represents the determination congruence $\Phi=\psi_{*} \wedge \psi_{+}$of $L$. In another instance, the $c c$-set $N(X)=\operatorname{Max}(X) \cap$ $\operatorname{Min}(X)$-the unique maximal $c c$-set which is an antichain-simultaneously represents both $\psi_{*} \vee \psi_{+}$and the finest congruence $\Psi$ such that the quotient $L / \Psi$ is a Boolean algebra. Therefore $\psi_{*} \vee \psi_{+}=\Psi$ as claimed in the introductory section.

Suppose that $X=V_{0} \cup V_{1} \cup N(X)$ is a decomposition as described by clause (2) of Theorem 1.1. Then $F_{i}=V_{i} \cup N(X)$ are closed for $i=0,1, F_{0} \supseteq \operatorname{Min}(X)$ and $F_{1} \supseteq \operatorname{Max}(X)$, so that $F_{0}$ represents a lattice congruence $\psi_{0} \leq \psi_{*}$ and $F_{1}$ represents a lattice congruence $\psi_{1} \leq \psi_{+}$. Moreover, $F_{0} \cap F_{1}=N(X)$ yields $\psi_{0} \vee \psi_{1}=\Psi=\psi_{*} \vee \psi_{+}$, and $\psi_{0} \wedge \psi_{1}=\omega$ because $F_{0} \cup F_{1}=X$. This shows that, in Theorem 1.1, (2) implies (3).

To verify the converse, let (3) of Theorem 1.1 hold, and let $F_{i} \subseteq X$ be the closed set that represents the congruence $\psi_{i}$ for $i=0,1$. Then $F_{0} \supseteq \operatorname{Min}(X)$ and $F_{1} \supseteq \operatorname{Max}(X)$ because $\psi_{0} \leq \psi_{*}$ and $\psi_{1} \leq \psi_{+}$. Furthermore, $F_{0} \cup F_{1}=X$ follows from $\psi_{0} \wedge \psi_{1}=\omega$, and $F_{0} \cap F_{1}=N(X)$ because $\psi_{0} \vee \psi_{1}=\Psi$. For $i=0,1$, the sets $V_{i}=X \backslash F_{1-i}$ are open and, together with $N(X)$, form a decomposition $X=V_{0} \cup V_{1} \cup N(X)$ of $X$ that obeys all requirements of clause (3) in Theorem 1.1 .

3. Proof of Theorem 1.1. To demonstrate that $(1) \Rightarrow(2)$ in Theorem 1.1 , let $f: Y \rightarrow X$ be the surjective $d p$-map dual to the inclusion homomorphism $L \subseteq K$. First we show that $f(y) \in \operatorname{Mid}(X)$ and $f^{-1}\{f(y)\}=\{y\}$ for every $y \in \operatorname{Mid}(Y)$.

If $y \in \operatorname{Mid}(Y)$ and $f(y)=a \in \operatorname{Ext}(X)$, then $\operatorname{Ext}(Y)$ and $\operatorname{Ext}(Y) \cup\{y\}$ are distinct $c c$-sets in $Y$ whose $f$-image is the $c c$-set $\operatorname{Ext}(X) \subseteq X$, which is impossible. Therefore $a=f(y) \in \operatorname{Mid}(X)$. If $f\left(y^{\prime}\right)=a$, then $y^{\prime} \in \operatorname{Mid}(Y)$ because $f$ is a $d p$-map. But then $\operatorname{Ext}(Y) \cup\{y\}$ and $\operatorname{Ext}(Y) \cup\left\{y^{\prime}\right\}$ are $c c$-sets mapped by $f$ onto the $c c$-set $\operatorname{Ext}(X) \cup\{a\}$, so that $y^{\prime}=y$. Since $f$ is surjective, we may assume that, up to the respective partial orders on these sets, $\operatorname{Mid}(X)=\operatorname{Mid}(Y)=M$.

Let $C \subseteq Y$ be a clopen decreasing set representing a core element of $K$, so that $\operatorname{Min}(Y) \subseteq C$ and $\operatorname{Max}(Y) \subseteq Y \backslash C$. Denoting $M_{0}=M \cap C$ and $M_{1}=M \backslash C$, we have $C=$ $M_{0} \cup \operatorname{Min}(Y)$ and $Y \backslash C=M_{1} \cup \operatorname{Max}(Y)$. Since $f$ is a continuous surjective $d p$-map that is the identity on $M$, the sets $f(C)=M_{0} \cup \operatorname{Min}(X)$ and $f(Y \backslash C)=M_{1} \cup \operatorname{Max}(X)$ are closed. Hence $V_{1}=X \backslash\left(M_{0} \cup \operatorname{Min}(X)\right)$ contains $\operatorname{Max}(X) \backslash \operatorname{Min}(X)$, the set $V_{0}=X \backslash\left(M_{1} \cup \operatorname{Max}(X)\right)$ contains $\operatorname{Min}(X) \backslash \operatorname{Max}(X)$, and $V_{0}$ and $V_{1}$ are open. Moreover, $V_{0} \cap V_{1}=\varnothing$ and $X \backslash\left(V_{0} \cup V_{1}\right)=\operatorname{Max}(X) \cap \operatorname{Min}(X)$. Therefore (2) holds.

$(2) \Rightarrow(1)$ : Assume (2). If $g: X^{\prime} \rightarrow X$ is the identity $d p$-map from Theorem 2.2 , then $g^{-1}\left(V_{0}\right)$ is open and decreasing, while $g^{-1}\left(V_{1}\right)$ is open and increasing.

Having used Theorem 2.2 in an initial extension step, we now assume that $\operatorname{Mid}(X)$ is an antichain, $V_{0}$ is open and decreasing, while $V_{1}$ is open and increasing. 
We define $Y$ as the disjoint union $Y=(X \backslash N(X)) \cup N(X) \times 2$, where elements of $X \bigvee(X)$ are incomparable to those from $N(X) \times 2$, the order on $X \backslash N(X) \subseteq Y$ coincides with that on $X$, and $(n, i)<\left(n^{\prime}, j\right)$ exactly when $n=n^{\prime}, i=0$ and $j=1$. A mapping $f: Y \rightarrow X$ defined by $f(n, 0)=f(n, 1)=n$ for all $n \in N(X)$ and $f(x)=x$ for all $x \in Y \backslash(X)$ thus preserves the order, and satisfies $\operatorname{Max}(f(y))=f(\operatorname{Max}(y))$ and $\operatorname{Min}(f(y))=$ $f(\operatorname{Min}(y))$ for all $y \in Y$. Furthermore, once we define a topology of $Y$ so that $f$ is continuous, then $Z \subseteq Y$ will be a $c c$-set if and only if $Z=f^{-1}(T)$ for some $c c$-set $T \subseteq X$.

To do this, recall that clopen sets that are increasing or decreasing form an open subbase of any Priestley space $X$.

Set $D_{i}=V_{i} \cup N(X) \times\{i\}$ for $i=0,1$. The topology $\sigma$ on $Y$ will have an open subbase

$$
\mathscr{S}=\left\{f^{-1}(C) \mid C \subseteq X \text { is clopen increasing or decreasing }\right\} \cup\left\{D_{11}, D_{1}\right\} .
$$

Observe that the sets $D_{0}, D_{1} \in \mathscr{S}$ are complementary and clopen, $\operatorname{Min}(Y) \subseteq D_{0}$ and $\operatorname{Max}(Y) \subseteq D_{1}$. Once we show that $Y$ is a $d p$-space, the set $D_{0}$ will thus represent a member of the core of $D(Y)$.

It is clear that $f: Y \rightarrow X$ is continuous.

Assume that $z \neq y$ in $Y$. Since $f: Y \rightarrow X$ collapses only pairs $(n, 0)<(n, 1)$ with $n \in N(X)$ and because $X$ is totally order disconnected, there is a clopen decreasing set $f^{-1}(C) \ni y$ such that $z \notin f^{-1}(C)$ whenever $(z, y) \neq((n, 1),(n, 0))$ for some $n \in N(X)$. But $(n, 0) \in D_{0} \not(n, 1)$ for the clopen decreasing set $D_{0} \in \mathscr{P}$; this shows that $Y$ is totally order disconnected.

To prove that $D_{0}$ is compact, select any subbasic open cover $\mathscr{B} \subseteq \mathscr{S}$ of $D_{0}$. Since $D_{0} \cap D_{1}=\varnothing$, we may assume that $D_{1} \notin \mathscr{B}$. Since there is nothing to prove when $D_{0} \in \mathscr{B}$, we have $\mathscr{B}=\left\{f^{-1}(C) \mid C \in \mathscr{C}\right\}$ for some system $\mathscr{C}$ of clopen sets that are increasing or decreasing in $X$. Since $f$ is surjective, $\mathscr{C}$ is an open cover of the closed set $f\left(D_{0}\right)=X \backslash V_{1}$. But then $f\left(D_{0}\right) \subseteq \bigcup \mathscr{C}^{\prime}$ for some finite $\mathscr{C}^{\prime} \subseteq \mathscr{C}$, and hence $D_{0}$ is covered by the finite subsystem $\left\{f^{-1}(C) \mid C \in \mathscr{C}^{\prime}\right\}$ of $\mathscr{B}$. Thus $D_{0}$ is compact. A similar argument applies to $D_{1}$, so that $Y=D_{0} \cup D_{1}$ is compact as well.

It remains to show that the Priestley space $Y$ is a $d p$-space. Let $S \subseteq Y$ be arbitrary. Then $f(\operatorname{Min}(\bar{S}))=\operatorname{Min}(f(\bar{S}))=\operatorname{Min} \overline{(f(S))}$ because $f$ has the $d p$-property and is a continuous map of compact Hausdorff spaces; similarly, $\overline{\operatorname{Min}(f(S))}=\overline{f(\operatorname{Min}(S))}=$ $f(\overline{\operatorname{Min}(S)})$. Moreover, $\operatorname{Min}(f(S))=\overline{\operatorname{Min}(f(S))}$ because $X$ is a $d p$-space (see Lemma 2.1), so that $f(\operatorname{Min}(\bar{S}))=f(\overline{\operatorname{Min}(S)})$. Thus if $y \in \operatorname{Min}(\bar{S})$, then $f(y)=f\left(y^{\prime}\right)$ for some $y^{\prime} \in$ $\overline{\operatorname{Min}(S)}$. But $\operatorname{Min}(Y)=D \cap f^{-1}(\operatorname{Min}(X))$ is closed, so that $y^{\prime} \in \operatorname{Min}(Y)$ as well. Since $f$ is one-to-one on $\operatorname{Min}(Y)$, it follows that $y=y^{\prime} \in \overline{\operatorname{Min}(S)}$. Therefore $\operatorname{Min}(\bar{S}) \subseteq \overline{\operatorname{Min}(S)}$ for any $S \subseteq Y$. Together with Lemma 2.1 and a dual argument, this shows that $Y$ is a $d p$-space. Hence (1) follows from (2).

The $d p$-space $Y$ constructed above contains no 4-element chain. From Adams and Beazer [1] it follows that the extension $K=D(Y)$ of $L=D(X)$ is congruence permutable.

Since $c c$-sets of $Y$ are in one-to-one correspondence to $c c$-sets of $X$, this completes the proof of Theorem 1.1.

To verify the remainder of Corollary 1.2 , it suffices to observe that, for any $d p$-space $X$ with $\operatorname{Ext}(X)=X$, the $d p$-space $Y$ satisfies $\operatorname{Ext}(Y)=Y$ as well.

4. Examples. For the sake of brevity, any $d p$-space satisfying 1.1(2) will be called 
extendable.

In this section we construct examples of non-extendable $d p$-spaces representing double Stone algebras, that is, members of the variety $\mathbf{S}$ generated by a 4-element chain. The two claims below suggest how to proceed.

Proposition 4.1. Any dp-space $X$ satisfying $\overline{\operatorname{Mid}(X)} \cap N(X)=\varnothing$ is extendable.

Proof. Since $X$ is a Priestley space such that $\operatorname{Max}(X)$ is closed, for every $a \in \overline{\operatorname{Mid}(X)} \cap \operatorname{Min}(X)$ there exists a clopen decreasing set $Y_{a}$ with $a \in Y_{a}$ and $Y_{a} \cap$ $\operatorname{Max}(X)=\varnothing$. Since $\operatorname{Mid}(X) \cap \operatorname{Min}(X)$ is closed and hence compact, for some finite set $I \subseteq \overline{\operatorname{Mid}(X)} \cap \operatorname{Min}(X)$ we have $\overline{\operatorname{Mid}(X)} \cap \operatorname{Min}(X) \subseteq Y=\bigcup\left\{Y_{a} \mid a \in I\right\}$; the set $Y$ is clopen, decreasing and $Y \cap \operatorname{Max}(X)=\varnothing$.

Furthermore, since both $Y$ and $\operatorname{Min}(X)$ are closed, for every $b \in \overline{\operatorname{Mid}(X)} \backslash Y$ there exists a clopen increasing set $Z_{b}$ such that $b \in Z_{b}$ and $Z_{b} \cap(Y \cup \operatorname{Min}(X))=\varnothing$. From the compactness of $\overline{\operatorname{Mid}(X)} \backslash Y$ follows the existence of a finite set $J \subseteq \overline{\operatorname{Mid}(X)} \backslash Y$ such that $\overline{\operatorname{Mid}(X)} \backslash Y \subseteq Z=\bigcup\left\{Z_{b} \mid b \in J\right\}$. Hence $Z$ is a clopen increasing set disjoint with $Y \cup \operatorname{Min}(X)$.

Therefore $V_{0}=X \backslash(Z \cup \operatorname{Max}(X))$ and $V_{1}=X \backslash(Y \cup \operatorname{Min}(X))$ are open and satisfy $V_{0} \cup V_{1}=X \backslash N(X)$. The set $V_{0}$ is decreasing and contains $\operatorname{Min}(X) \backslash \operatorname{Max}(X)$, while $V_{1}$ is increasing and contains $\operatorname{Max}(X) \backslash \operatorname{Min}(X)$. From $\operatorname{Mid}(X) \subseteq Y \cup Z$ it follows that $V_{0} \cap V_{1}=$ $\varnothing$, so that the condition (2) of Theorem 1.1 is satisfied.

As a consequence of Proposition 4.1, the set $\operatorname{Mid}(X)$ must be infinite for any non-extendable $d p$-space $X$. Furthermore, because $\operatorname{Min}(\operatorname{Mid}(X)) \subseteq \operatorname{Min}(X) \backslash \operatorname{Max}(X)$, from Lemma 2.1 it follows that $N(X)$ must intersect the closure of $\operatorname{Min}(X) \backslash \operatorname{Max}(X)$ and, dually, also the closure of $\operatorname{Max}(X) \backslash \operatorname{Min}(X)$, in the same set. In addition, since the sets $X \backslash \operatorname{Max}(X)$ and $X \backslash \operatorname{Min}(X)$ are open in any $d p$-space $X$, the set $\overline{\operatorname{Mid}(X)}$ of a non-extendable $d p$-space intersects both $\operatorname{Max}(X) \backslash \operatorname{Min}(X)$ and $\operatorname{Min}(X) \backslash \operatorname{Max}(X)$.

Somewhat surprising is the fact that a non-extendable $d p$-space must satisfy yet another requirement.

Proposition 4.2. Any dp-space $X$ with countable $\operatorname{Min}(X) \backslash \operatorname{Max}(X)$ or countable $\operatorname{Max}(X) \backslash \operatorname{Min}(X)$ is extendable.

Proof. Let $\mathbb{N}$ be the set of natural numbers and let $\operatorname{Min}(X) \backslash \operatorname{Max}(X)=\left\{a_{i} \mid i \in \mathbb{N}\right\}$. Arguing inductively, for each $i \in \mathbb{N}$ we find a clopen decreasing set $A_{i}$ with $a_{i} \in A_{i}$ and $A_{i} \cap \operatorname{Max}(X)=\varnothing$, and such that for every $j \in \mathbb{N}$ with $j \leq i$ either $A_{i}=A_{j}$ or $A_{i} \cap A_{j}=\varnothing$. Set $B_{i}=\left[A_{i}\right) \backslash A_{i}$ for each $i \in \mathbb{N}$. Since $\left[A_{i}\right]$ is clopen, it follows that $B_{i}$ is a clopen increasing set, and that $B_{i} \cap A_{j}=\varnothing$ for all $i, j \in \mathbb{N}$.

Define $V_{0}=\bigcup\left\{A_{i} \mid i \in \mathbb{N}\right\}$ and $V_{1}=\bigcup\left\{B_{i} \mid i \in \mathbb{N}\right\}$. The sets $V_{0}$ and $V_{1}$ then satisfy (2) of Theorem 1.1. The proof is dual when $\operatorname{Max}(X) \backslash \operatorname{Min}(X)$ is countable.

To design examples of non-extendable spaces, we first define an auxiliary Stone space $(S, \sigma)$ which will serve as their initial building block. We proceed as follows.

Let $I$ be any infinite set, and let $\mathscr{P}$ be an infinite family of infinite subsets of $I$. We say that the family $\mathscr{P}$ is almost disjoint provided $P \cap P^{\prime}$ is finite whenever $P, P^{\prime} \in \mathscr{P}$ are distinct.

For any infinite system $\mathscr{P}$ of infinite subsets of $I$, set $Z=Z(\mathscr{P})=\bigcup \mathscr{P}$, and

$$
S(\mathscr{P})=I \cup\left\{c^{P} \mid P \in \mathscr{P}\right\} \cup\{c\}
$$


where the union is disjoint. Let $\sigma$ be the family of all subsets $V$ of $S=S(\mathscr{P})$ such that

(1) if $c^{r} \in V$, then the set $P \backslash V$ is finite, and

(2) if $c \in V$, then

(a) the set $\left\{c^{P} \mid P \in \mathscr{P}\right\} \backslash V$ is finite,

(b) the set $I \backslash(Z \cup V)$ is finite, and

(c) there is a finite $\mathscr{P}^{\prime} \subset \mathscr{P}$ such that $Z \backslash \cup \mathscr{P}^{\prime} \subseteq V$.

It is routine to verify that $\varnothing, S \in \sigma$, and that the collection $\sigma$ is closed under finite intersections and arbitrary unions. Hence $\sigma$ is the family of all open sets of a topological space $(S, \sigma)$ in which the singleton $\left\{c^{P}\right\}$ compactifies $P \subseteq I$ for each $P \in \mathscr{P}$, and $S$ is a one-point compactification of $S \backslash\{c\}$; in particular, the topological space $(S, \sigma)$ is compact. Next we aim to show that, if $\mathscr{P}$ is an almost disjoint family, then any two distinct members of $(S, \sigma)$ can be separated by clopen sets. This will prove that the (unordered) space $(S, \sigma)$ is a Stone space, that is, the Priestley dual of a Boolean algebra.

First we note that $I$ is a discrete subspace of $(S, \sigma)$ : obviously, $\{i\} \in \sigma$ for every $i \in I$, and it is easy to verify that $S \backslash\{i\}$ satisfies (1) and (2).

Secondly, for any $P \in \mathscr{P}$, let $A \subseteq S$ be a set such that $c^{P} \in A \subseteq P \cup\left\{c^{P}\right\}$ and $P \backslash A$ is finite. Clearly, $A \in \sigma$ and $S \backslash A$ satisfies (2). To see that $S \backslash A$ satisfies also (1), we note that $Q \in \mathscr{P}$ and $c^{Q} \in S \backslash A$ imply $Q \neq P$; but then $Q \backslash(S \backslash A) \subseteq Q \cap P$ is finite because the family $\mathscr{P}$ is almost disjoint. Hence $S \backslash A \in \sigma$, as claimed.

Let $s, t$ be distinct elements of $S$. If $s \in I$, then $\{s\}$ is clopen and separates $s$ from $t$; if $s=c^{Q}$ for some $Q \in \mathscr{P}$, then the clopen set $Q \cup\{s\}$ separates $s$ from any $t \in \cup\left\{c^{P} \mid P \neq\right.$ $Q\} \cup\{c\}$. This shows that $(S, \sigma)$ is totally (order) disconnected, and completes the proof of the claim below.

Lemma 4.3. The space $(S, \sigma)$ is a Stone space such that $I \subset S$ is its discrete subspace, $S$ is a one-point compactification of $S \backslash\{c\}$ and, for any $P \in \mathscr{P}$, the subspace $P \cup\left\{c^{p}\right\}$ is $a$ one-point compactification of $P$.

Write $T=Z \cup\left\{c^{p} \mid P \in \mathscr{P}\right\} \cup\{c\}$, and define an ordered topological space $Y$ as a (disjoint) union

$$
Y=(S \times\{0,2\}) \cup(T \times\{1\})
$$

such that $(s, i) \leq\left(s^{\prime}, i^{\prime}\right)$ if and only if $s=s^{\prime}$ and $i \leq i^{\prime}$. Thus the partial order of $Y$ is a disjoint union of 3 -element chains $\{t\} \times\{0,1,2\}$ with $t \in T$ and 2-element chains $\{j\} \times\{0,2\}$ with $j \in J=I \backslash Z$.

Since $S$ and $T$ are compact, so is $Y$. A subset $B$ of $Y$ is clopen exactly when $B_{i}=\{s \in S \mid(s, i) \in B\}$ is clopen in $S$ for each $i \in\{0,1,2\}$. Therefore $(I \times\{0,2\}) \cup$ $(Z \times\{1\})$ is discrete, and $B \subseteq\left(P \cup\left\{c^{P}\right\}\right) \times\{i\}$ is clopen whenever $c^{P} \in B_{i}$ and $P \backslash B_{i}$ is finite; it follows that $Y$ is totally order disconnected. If $D \subseteq Y$ is clopen and decreasing, then $[D]=\left[D_{0} \times\{0\}\right)$; since $D_{0}$ is clopen in $S$, the set $[D]$ is clopen in $Y$. Together with a dual observation, this shows that $Y$ is a $d p$-space.

Select infinite subfamilies $\mathscr{Q}$ and $\mathscr{R}$ of $\mathscr{P}$ such that $\mathscr{Q} \cup \mathscr{R}=\mathscr{P}$ and $\mathscr{2} \cap \mathscr{R}=\varnothing$. Let $\theta$ be an equivalence on $Y$ whose nontrivial classes are all pairs $\left\{\left(c^{Q}, 1\right),\left(c^{Q}, 2\right)\right\}$ with $Q \in \mathcal{Q}$, all pairs $\left\{\left(c^{R}, 0\right),\left(c^{R}, 1\right)\right\}$ with $R \in \mathscr{R}$, and the triple $\{(c, 0),(c, 1),(c, 2)\}$. Set $X=Y / \theta$, and let $f: Y \rightarrow X$ be the surjective mapping with $\operatorname{Ker}(f)=\theta$. Write $f\left(c^{Q}, 1\right)=f\left(c^{Q}, 2\right)=$ $c_{2}^{Q}$ and $f\left(c^{Q}, 0\right)=c_{0}^{Q}$ for all $Q \in \mathscr{Q}, f\left(c^{R}, 0\right)=f\left(c^{R}, 1\right)=c_{0}^{R}$ and $f\left(c^{R}, 2\right)=c_{2}^{R}$ for all $R \in \mathscr{R}$, $f(c, 0)=f(c, 1)=f(c, 2)=c$, and $f(s, i)=(s, i)$ for all other $(s, i) \in Y$. 
When equipped with the quotient order $\leq$ and the quotient topology $\tau$ inherited from $Y$, the space $X=X(I, \mathscr{Q}, \mathscr{R})=(X, \tau, \leq)$ is compact, and the mapping $f: Y \rightarrow X$ is continuous and order preserving. Since $\operatorname{Max}(y)$ and $\operatorname{Min}(y)$ are singletons for every $y \in Y$, and because $f$ only collapses an element of $Y$ with an extremal element comparable to it, $f$ has the $d p$-property, that is, $f(\operatorname{Max}(y))=\operatorname{Max}(f(y))$ and $f(\operatorname{Min}(y))=\operatorname{Min}(f(y))$ for every $y \in Y$.

Described less formally, $(X, \tau, \leq)$ is a disjoint union of 3-element chains $\{z\} \times$ $\{0,1,2\}$ with $z \in Z$, of 2-element chains $\left\{c_{0}^{P}, c_{2}^{P}\right\}$ with $P \in \mathscr{P}$ and $\{j\} \times\{0,2\}$ with $j \in I Z$, and of a singleton chain $\{c\}$. All these chains are maximal. The subspace $(I \times\{0,2\}) \cup$ $(Z \times\{1\})$ is discrete, $\left\{c_{0}^{Q}\right\}$ compactifies $Q \times\{0\}$ and $\left\{c_{2}^{Q}\right\}$ compactifies $Q \times\{1,2\}$ for $Q \in \mathcal{Q}$, while $\left\{c_{0}^{R}\right\}$ compactifies $R \times\{0,1\}$ and $\left\{c_{2}^{R}\right\}$ compactifies $R \times\{2\}$ for each $R \in \mathscr{R}$ and, finally, $X$ is a one-point compactification of $X \backslash\{c\}$.

To show that $X$ is totally order disconnected, for any $b \neq a$ in $X$, we must exhibit a clopen decreasing set $A \subseteq X$ such that $a \in A$ and $b \notin A$. If $a=f(i, j)$ for some $i \in I$ and $j \in\{0,1,2\}$, then we choose the finite set $A=(a]$; when $b=f(i, j)$, we select $A=X \backslash b)$.

We may thus assume that $a, b \in C=\left\{c_{j}^{P} \mid P \in \mathscr{P}\right.$ and $\left.j \in\{0,2\}\right\} \cup\{c\}$. Let $a=c_{2}^{\prime}$ for some $P \in \mathscr{P}$. Then $b \neq c_{0}^{P}$ and we choose $A=(P \times\{0,1,2\}) \cup\left\{c_{0}^{P}, c_{2}^{P}\right\}$. Since $A$ is also increasing, its complement $A^{\prime}=X \backslash A$ separates any $a \in C \backslash\left\{c_{0}^{P}, c_{2}^{P}\right\}$ from any $b \in\left\{c_{0}^{P}, c_{2}^{P}\right\}$. The remaining case is that of $a=c_{0}^{P}$ and $b=c_{2}^{P}$ : for $P \in \mathscr{Q}$ we choose $A=(P \times\{0\}) \cup$ $\left\{c_{0}^{P}\right\}$, and for $P \in \mathscr{R}$ we select $A=(P \times\{0,1\}) \cup\left\{c_{0}^{P}\right\}$.

Therefore $X$ is the Priestley dual of a distributive $(0,1)$-lattice and $f$ is the Priestley dual of a lattice $(0,1)$-homomorphism that preserves all existing pseudocomplements and dual pseudocomplements.

If $D \subseteq X$ is clopen and decreasing, then $f^{-1}(D)$ is clopen and decreasing because $f$ is continuous and order preserving. Since $Y$ is a $d p$-space, the set $\left[f^{-1}(D)\right)$ is clopen in $Y$. If $f(y) \geq d \in D$, then $f(y) \geq m_{d}$ for the minimal element $m_{d} \leq d$; the $d p$-property of $f$ implies that $m_{d}=f\left(n_{y}\right)$ for the minimal element $n_{y} \leq y$ of $Y$. Hence $f^{-1}([D)) \subseteq\left[f^{-1}(D)\right)$, and $f^{-1}([D))=\left[f^{-1}(D)\right)$ follows because the reverse inclusion is trivial. Since $X$ has the quotient topology of $Y$, we conclude that $[D) \subseteq X$ is clopen. Together with a dual argument, this shows that $X=X(I, 2, \mathscr{R})$ is a $d p$-space representing a subalgebra of the Priestley dual of $Y$. The partial order of $X$ is a disjoint union of chains of length at most three, so that $X$ is a Priestley dual of a double Stone algebra.

This demonstrates the claim below.

Lemma 4.4. Let $I$ be an infinite set and $\mathscr{P}$ an infinite almost disjoint family of infinite subsets of $I$. Then any decomposition $\mathscr{P}=\mathscr{Q} \cup \mathscr{R}$ of $\mathscr{P}$ into infinite subsystems 2 and $\mathscr{R}$ gives rise to a dp-space $X=X(I, \mathscr{Q}, \mathscr{R})$ dual to a double Stone algebra.

We are now ready to produce examples of non-extendable $d p$-spaces. These will be of the form $X=X(I, \mathscr{Q}, \mathscr{R})$ for some decomposition $\mathscr{P}=\mathscr{Q} \cup \mathscr{R}$ of a suitable almost disjoint system $P$ on an infinite set $I$.

Proposition 4.5. If $\alpha \leq \beta$ are cardinals, $\alpha$ uncountable, then there exists a double Stone algebra whose Priestley dual $X$ is non-extendable and satisfies $|\operatorname{Mid}(X)|=\alpha$ and $|\operatorname{Max}(X)|=|\operatorname{Min}(X)|=\beta$.

Proof. Let $K$ be a set with $|K|=\alpha$, and let $I \supseteq K \times K$ have cardinality $\beta$. For any $k, l \in K$ write $Q_{k}=\{k\} \times K$ and $R_{l}=K \times\{l\}$. If $\mathscr{Q}=\left\{Q_{k} \mid k \in K\right\}$ and $\mathscr{R}=\left\{R_{l} \mid l \in K\right\}$, 
then $\mathscr{P}=\mathscr{Q} \cup \mathscr{R}$ is an almost disjoint system on $I$ and $Z=K \times K$. We need to show that the $d p$-space $X=X(I, 2, \mathscr{R})$ violates $1.1(2)$.

Suppose that $V_{0}$ and $V_{1}$ are open sets such that $V_{0} \supseteq \operatorname{Min}(X) \backslash \operatorname{Max}(X)=\operatorname{Min}(X) \backslash\{c\}$ and $V_{1} \supseteq \operatorname{Max}(X) \backslash\{c\}$. Since $c_{0}^{R} \in V_{0}$ for every $R \in \mathscr{R}$ and because $c_{0}^{R}$ compactifies $R \times\{1\}$, the set $R, \times\{1\} \backslash V_{0}$ is finite for every $l \in K$. Similarly, the set $Q_{k} \times\{1\} \backslash V_{1}$ is finite for every $k \in K$.

For any $l \in K$, set $K_{l}=\left\{k \in K \mid(k, l, 1) \in V_{0}\right\}$. Then $K \backslash K_{l}=\left\{k \in K \mid(k, l, 1) \notin V_{0}\right\}$ is finite because $\left\{c_{0}^{R_{1}}\right\}$ compactifies the discrete subspace $R_{l} \times\{1\}$. Select any countably infinite subset $L$ of $K$. Then $M=\bigcup\left\{K \backslash K_{l} \mid l \in L\right\}$ is countable, and $\cap\left\{K_{l} \mid l \in L\right\}=$ $K \backslash M \neq \varnothing$ follows from the fact that $|K|=\alpha$ is uncountable. Select and fix $\forall \times\{1\}$ some $k \in \bigcap\left\{K_{l} \mid l \in L\right\}$. Then $\{k\} \times L \times\{1\} \subseteq V_{0}$ and, if $V_{0} \cap V_{1}=\varnothing$, then the set $Q_{k} \backslash V_{1} \supseteq$ $\{k\} \times L \times\{1\}$ is infinite, in contradiction to the fact that $\left\{c_{2}^{Q_{k}}\right\} \subseteq V_{1}$ compactifies the discrete subspace $Q_{k} \times\{1\}$. Hence $X$ fails to satisfy $1.1(2)$.

Proposition 4.6. For any cardinal $\beta \geq 2$ in' there exists a double Stone algebra whose Priestley space $X$ is non-extendable and such that $|\operatorname{Mid}(X)|=\aleph_{0}$ and $|\operatorname{Max}(X)|=$ $|\operatorname{Min}(X)|=\beta$.

Proof. Let $\mathbb{N}$ be the set of all positive integers. To construct a non-extendable $d p$-space $X=X(I, \mathscr{2}, \mathscr{R})$, we begin with some infinite collection 2 of infinite pairwise disjoint subsets of $\mathbb{N}$, select a maximal almost disjoint system $\mathscr{P} \supset \mathscr{Q}$ of subsets of $\mathbb{N}$, and set $\mathscr{R}=\mathscr{P} \backslash \mathscr{2}$. Then $\mathscr{R}$ has at most $2^{\kappa_{1 \prime}}$ elements. Set $Z=\mathbb{N}$ and let $I \supseteq Z$ be a set of cardinality $\beta$. The $d p$-space $X=X(I, \mathscr{2}, \mathscr{R})$ then satisfies all cardinality requirements.

To show that $X$ is non-extendable, suppose that open sets $V_{0} \supseteq \operatorname{Min}(X) \backslash\{c\}$ and $V_{1} \supseteq \operatorname{Max}(X) \backslash\{c\}$ form a decomposition of $X \backslash\{c\}$. Set $J=\left\{j \in \mathbb{N} \mid(j, 1) \in V_{1}\right\}$.

Assume first that $R \cap J$ is finite for every $R \in \mathscr{R}$. Since $c_{2}^{Q} \in V_{1}$ compactifies the discrete subspace $Q \times\{1\}$, the set $Q \times\{1\} \backslash V_{1}$ is finite and we may thus choose some $j_{Q} \in Q$ such that $\left(j_{Q}, 1\right) \in V_{1}$ for each $Q \in \mathscr{Q}$. The set $R^{\prime}=\left\{j_{Q} \mid Q \in \mathscr{Q}\right\}$ is infinite, $R^{\prime} \cap Q=\left\{j_{Q}\right\}$ for every $Q \in \mathscr{2}$, and $R^{\prime} \cap R \subseteq J \cap R$ is finite for each $R \in \mathscr{R}$. Hence $\mathscr{P} \cup\left\{R^{\prime}\right\}$ is an almost disjoint system, in contradiction to the maximality of $\mathscr{P}$.

Therefore $R \cap J$ is infinite for some $R \in \mathscr{R}$, that is, the set $(R \times\{1\}) \cap V_{1}$ is infinite. Since $c_{0}^{R} \in V_{0}$ compactifies the discrete subspace $R \times\{1\}$, the set $R \times\{1\} \backslash V_{0}$ must be finite. But then $(R \times\{1\}) \cap V_{0} \cap V_{1}$ is an infinite set. This contradiction shows that $X$ fails to satisfy $1.1(2)$.

Remark. Denoting $A=\operatorname{Min}(X) \backslash \operatorname{Max}(X), B=\operatorname{Mid}(X)$ and $C=\operatorname{Max}(X) \backslash \operatorname{Min}(X)$, we can summarize the result of this section as follows. Any $d p$-space $X$ with finite $B$, or countable $A$ or $C$ is extendable. On the other hand, there are examples of non-extendable spaces with arbitrarily large uncountable $A, B$ and $C$, and an example in which $B$ is countable and $A, C$ whose cardinality is at least that of the continuum. Even though this covers all possible cases when the continuum hypothesis is assumed, we are unaware of any non-extendable $d p$-space $X$ with countable $B=\operatorname{Mid}(X)$, other than that provided by Proposition 4.6.

ACKnowledgement. The support of the NSERC of Canada is gratefully acknowledged by the second author. 


\section{REFERENCES}

1. M. E. Adams and R. Beazer, Congruence properties of distributive double $p$-algebras, Czech. Math. J., to appear.

2. R. Beazer and J. Sichler, Congruence permutable extensions of distributive double p-algebras, Proc. Edinburgh Math. Soc., to appear.

3. B. A. Davey, Subdirectly irreducible distributive double $p$-algebras, Algebra Universalis 8 (1978), 73-88.

4. T. Katriňák, Congruence extension property for distributive double $p$-algebras, Algebra Universalis 4 (1974), 273-276.

5. V. Koubek and J. Sichler, Categorical universality of regular double p-algebras, Glasgow Math. J. 32 (1990), 329-340.

6. H. A. Priestley, Representation of distributive lattices by means of ordered Stone spaces, Bull. London Math. Soc. 2 (1970), 186-190.

7. H. A. Priestley, Ordered topological spaces and the representation of distributive lattices, Proc. London Math. Soc. 24 (1972), 507-530.

8. H. A. Priestley, Ordered sets and duality for distributive lattices, Ann. Discrete Math. 23 (1984), 36-90.

9. J. Varlet, A regular variety of type $\langle 2,2,1,1,0,0\rangle$, Algebra Universalis 2 (1972), 218-223.

\section{MFF KU}

Malostranské NÁM. 25

11800 Praha 1

Czech Republic
Department of Mathematics

UNIVERSITY OF MANITOBA

WinNiPEG, ManitoBa

CANADA R3T 2N2 